BMJ Nutrition,

Prevention \& Health

\title{
Nutritional parameters and outcomes in patients admitted to intensive care with COVID-19: a retrospective single-centre service evaluation
}

\author{
Timothy Eden, ${ }^{1,2}$ Shane McAuliffe (10 , , ${ }^{2,3}$ Dominic Crocombe, ${ }^{2}$ Jonathan Neville, ${ }^{1}$ \\ Sumantra Ray ${ }^{2,4,5,6}$
}

Background COVID-19 is an inflammatory syndrome caused by novel coronavirus SARS-CoV-2. Symptoms range from mild infection to severe acute respiratory distress syndrome (ARDS) requiring ventilation and intensive care. At the time of data collection, UK cases were around 300000 with a fatality rate of $13 \%$ necessitating over 10000 critical care admissions; now there have been over 4 million cases. Nutrition is important to immune function and influences metabolic risk factors such as obesity and glycaemic control, as well as recovery from acute illnesses. Poor nutritional status is associated with worse outcomes in ARDS and viral infections, yet limited research has assessed pre-morbid nutritional status and outcomes in patients critically unwell with COVID-19.

Objectives Investigate the effect of body mass index (BMI), glycaemic control and vitamin D status on outcomes in adult patients with COVID-19 admitted to an intensive care unit (ICU).

Methods Retrospective review of all patients admitted to a central London ICU between March and May 2020 with confirmed COVID-19. Electronic patient records data were analysed for patient demographics; comorbidities; admission BMl; and serum vitamin D, zinc, selenium and haemoglobin $\mathrm{A} 1 \mathrm{c}(\mathrm{HbA1c})$ concentrations. Serum vitamin $\mathrm{D}$ and $\mathrm{HbA1c}$ were measured on admission, or within 1 month of admission to ICU. Primary outcome of interest was mortality. Secondary outcomes included time intubated, ICU stay duration and ICU-related morbidity. Results Seventy-two patients; 54 (75\%) men, mean age $57.1( \pm 9.8)$ years, were included. Overall, mortality was $24(33 \%)$. No significant association with mortality was observed across BMI categories. In the survival arm admission, $\mathrm{HbA} 1 \mathrm{c}$ (mmol/mol) was lower, 50.2 vs 60.8 , but this was not statistically significant. Vitamin $D$ status did not significantly associate with mortality $(p=0.131)$. However, $32 \%$ of patients with low vitamin D (<25 IU/L) died, compared with $13 \%$ of patients with vitamin $D$ levels $>26 \mathrm{IU} / \mathrm{L}$. Serum zinc and selenium, and vitamin $\mathrm{B}_{12}$ and folate levels were measured in $46 \%$ and $26 \%$ of patients, respectively.

Discussion/conclusion Increased adiposity and deranged glucose homeostasis may potentially increase risk of COVID-19 infection and severity, possibly relating to impaired lung and metabolic function, increased proinflammatory and prothrombotic mechanisms. Vitamin

\section{What this paper adds}

- This snapshot audit in a London ICU from the first peak of the COVID-19 pandemic adds to the body of evidence associating overweight/obesity as a risk factor of disease severity and mortality in COVID-19.

- Further data suggesting baseline vitamin D status may influence severity of COVID-19 as higher mortality was observed in individuals with vitamin $D$ deficiency ( $<25 \mathrm{nmol} / \mathrm{L})$.

- This highlights baseline nutritional parameters that are easily obtainable in the critical care setting and suggests additional nutritional markers worthy of further research.

D deficiency may also associate with poorer outcomes and mortality, supporting a possible role of vitamin D in immune function specific to pulmonary inflammation and COVID-19 pathophysiology. There are plausible associations between raised BMI, glycaemic control, vitamin D status and poor prognosis, as seen in wider studies; however, in this service evaluation audit during the first wave of the pandemic in the UK, with a limited data set available for this analysis, the associations did not reach statistical significance. Further research is needed into specific nutritional markers influencing critical care admissions with COVID-19.

\section{INTRODUCTION}

As COVID-19 cases continue to rise worldwide, so does the role of nutritional factors on the agendas of government, public health, primary and secondary healthcare bodies. One aspect of clinical importance is the role of nutrition in critical care, particularly how specific baseline parameters of nutritional status may influence illness severity, morbidity and mortality. At the time of data collection and analysis in September 2020, UK cases have exceeded 300000 with a fatality rate of $\sim 13 \%$ necessitating $>10000$ critical care admissions. ${ }^{1}$ It is therefore paramount we learn from previous and current practice to 
improve our understanding and clinical management of COVID-19 in the wake of the continuing pandemic if we are to minimise future morbidity and mortality.

As SARS-CoV-2 is a novel coronavirus, it is not yet fully understood which specific nutritional parameters are most impactful on disease severity and outcomes. However, it is well established that nutrition plays a key role in immune function and several macronutrients and micronutrients are required for key physiological processes during infection. ${ }^{2}$ Globally, clinical data collection has highlighted certain nutritional factors that may influence outcomes of COVID-19. ${ }^{3}$ However, there are limited data published that relate to the nutritional status of patients on admission to critical care units, thus we present data from this context.

\section{Audit rationale and selected parameters}

The rationale for this audit was to present data on nutritional parameters of patients with COVID-19 from a real-world intensive care unit (ICU) setting. We wanted to determine whether there were any clear associations between mortality and nutritional parameters that (1) are commonly measured in clinical practice, and (2) have been identified as possible risk factors for worse outcomes in the care of inpatients with COVID-19. Parameters of particular interest were obesity, glycaemic control and vitamin D status.

\section{Obesity: body mass index}

It has been demonstrated that higher body mass index (BMI) and obesity are associated with poorer outcomes in COVID-19. ${ }^{4}$ One large cohort study examined the factors associated with COVID-19-related hospital deaths in the linked electronic health records of 17 million adult National Health Service (NHS) patients. It found that the risk of death increased with the degree of obesity: fully adjusted HR was 1.05 for obese class I, increasing to 1.92 for obese class III $\left(\right.$ BMI $\left.\geq 40 \mathrm{~kg} / \mathrm{m}^{2}\right) .^{5}$ A key consideration in critical care is that increased body mass adversely impacts pulmonary function by reducing expiratory reserve volume, functional capacity and respiratory system compliance. ${ }^{6}$ These factors, in addition to technical airway difficulties associated with obesity, are deleterious for patients who require mechanical ventilation and precipitate further respiratory complications. Increased adiposity is also associated with increased proinflammatory cytokines that have been implicated in the pathogenesis of COVID-19. ${ }^{2}$

\section{Type 2 diabetes and glycaemic control: haemoglobin A1c}

Other established risk factors for worse COVID-19 outcomes include cardiometabolic diseases: type 2 diabetes mellitus (T2DM), hypertension and dyslipidaemia. ${ }^{57}$ A whole population study of England during the first peak of the pandemic indicated that people with T2DM had a twofold increased risk of inpatient COVID19-related deaths relative to people without diabetes. ${ }^{8}$ This study also indicated significance when adjusting for cardiovascular disease and T2DM that remained independent risk factors for mortality when further adjusting for age, ethnicity and social deprivation. Although glycaemic control targets have remained controversial in the critical care setting, it is established that baseline insulin sensitivity and subsequent glycaemic control can influence mortality in the general population. ${ }^{9}$ It could therefore be speculated that individuals with T2DM presenting with COVID-19 may benefit from tighter glycaemic control.

\section{Vitamin D status: 25-hydroxyvitamin D}

Another area of interest is the role of micronutrients and how individual baseline levels may impact morbidity and mortality. Vitamin D status has attracted much attention as a secosteroid with biological roles in immune function and anti-inflammatory, antifibrotic and antioxidant effects. ${ }^{10}$ Vitamin D insufficiency (defined as serum levels $25-50 \mathrm{nmol} / \mathrm{L})$ and deficiency $(<25 \mathrm{nmol} / \mathrm{L})$ across the UK are estimated at approximately $23 \%$ in people aged 19-64 years increasing in prevalence in the months of January-March to $40 \% .{ }^{11}$ It is recognised that vitamin D status across the UK significantly reduces in the winter months due to the reliance of skin synthesis and the lack of required ultraviolet B exposure from sunlight. National and localised lockdowns have led to reduced outdoor activity and subsequently less sun exposure in many groups thus likely further impacting vitamin D levels. Across Europe, ecological studies indicate an inverse correlation between estimates of vitamin D status and COVID-19 incidence and mortality. ${ }^{12}$

An association between vitamin $\mathrm{D}$ status and respiratory tract infections, tuberculosis and chronic obstructive pulmonary disease is long-established and supplementation in individuals with low vitamin D levels has improved outcomes in interventional studies. ${ }^{13}$ There is a plethora of mechanistic pathways linking vitamin $\mathrm{D}$ to pulmonary immune function that could have a role in COVID-19 pathophysiology. While robust interventional trials are awaited in this area, given the high prevalence of suboptimal vitamin D levels in the general population, and the additional risk during autumn and winter months, exploring the link between baseline vitamin D status and COVID-19 severity could further our understanding of how to optimally supplement in high-risk groups.

\section{Other micronutrients}

Several other micronutrients are essential to healthy immune responses and many may have a role in COVID-19 outcomes, particularly in deficiency. The practice of measuring serum levels of other micronutrients varies greatly between individuals. Nonetheless, for completeness of data regarding nutritional parameters, we also wanted to present recorded levels of zinc, selenium, magnesium, vitamin $\mathrm{B}_{12}$ and folate for all patients in whom these were measured. 


\section{Aims}

We aimed to investigate the relationship between BMI, glycaemic control and vitamin D status, respectively, on outcomes in patients admitted to the ICU with COVID-19 pneumonia. Primary measure was mortality. Secondary measures included time intubated, ICU stay duration and ICU-related morbidity.

\section{Methods}

We undertook a retrospective review of all patients admitted to a West London ICU between 18 March and 18 May 2020 with confirmed COVID-19, as confirmed clinically and by a positive oropharyngeal or nasopharyngeal SARS-CoV-2 PCR test. No patients were excluded from analysis.

Data were extracted from the electronic patient database. The following data were collected: patient demographics; comorbidities; BMI on admission (using ICU bedscale or patient database); serum vitamin D concentration; mean weekly random plasma glucose and plasma haemoglobin A1c (HbA1c). Serum vitamin D concentration and $\mathrm{HbAlc}$ were taken on admission, or within 1 month of admission to ICU. The serum $\mathrm{C}$ reactive protein (CRP) was also recorded at the same time point as vitamin $\mathrm{D}$. The requirement for insulin, steroid, antibiotic, intravenous water-soluble vitamin concentrate (the preparation used in this department was Pabrinex, which contains thiamine, riboflavin, pyridoxine hydrochloride, ascorbic acid, nicotinamide and glucose) and renal replacement therapy at any time point during the admission was also recorded. The primary outcome measure was mortality. Secondary outcome measures included the time intubated, the length of ICU stay and ICU-related morbidity.

Statistical analysis was undertaken using SPSS V.24 (IBM). Statistical significance was set as $\mathrm{p}<0.05$. Continuous parametric data sets were compared by the t-test, and non-parametric data sets by the Mann-Whitney U test. Categorical data were compared using $\mathrm{X}^{2}$ test. Data are presented as mean $( \pm \mathrm{SD})$ or median $(\mathrm{IQR})$.

\section{RESULTS}

In total, 72 patients were included in the service evaluation (see online supplemental table 1 for summary of demographics). Of these, 48 survived $(68 \%)$ and were discharged from ICU. Fifty-four patients $(75 \%)$ were men, and mean age was $57.1( \pm 9.8)$ years. Nineteen patients had no comorbidities documented on admission. The most common comorbidities were hypertension $(40 \%)$, T2DM (26\%) and chronic kidney disease (20\%). Six patients $(8 \%)$ received oral nutrition only during their ICU admission, 58 (81\%) required enteral nutrition (57 via nasogastric tube (NGT), 1 gastrostomy), 3 (4\%, including 2 of those who also required NGT) required parenteral nutrition, and these data were not recorded for 7 patients $(10 \%)$.

\begin{tabular}{|lccc|}
\hline Table 1 & \multicolumn{4}{c|}{ BMI categories and primary outcome of survival } \\
\hline BMI, $\mathbf{~ k g / \mathbf { m } ^ { 2 }}$ & $\begin{array}{l}\text { Survived, } \\
\mathbf{n = 4 8}\end{array}$ & Died (n=24) & P value \\
\hline $\mathbf{1 8 . 5 - 2 4 . 9}(\%)$ & $8(61.5)$ & $5(38.4)$ & 0.589 \\
$\mathbf{2 5 . 0 - 2 9 . 9 ( \% )}$ & $22(64.7)$ & $12(35.3)$ & \\
$\mathbf{3 0 . 0 - 3 4 . 9}(\%)$ & $10(71.4)$ & $4(28.6)$ & \\
$\mathbf{3 5 . 0 - 3 9 . 9 ( \% )}$ & $4(57.1)$ & $3(42.9)$ & \\
$\mathbf{2 4 0 . 0}(\%)$ & $4(100.0)$ & $0(0.0)$ & \\
\hline
\end{tabular}

BMI, body mass index.

\section{Body mass index}

Mean BMI was $29.5 \mathrm{~kg} / \mathrm{m}^{2}(\mathrm{SD} \pm 5.7)$ for the survival group and $28.2 \mathrm{~kg} / \mathrm{m}^{2}(\mathrm{SD} \pm 4.8)$ for the non-survivors. No patients were admitted with a BMI $<18.5 \mathrm{~kg} / \mathrm{m}^{2}$. No significant difference in mortality was observed across different BMI categories, $\mathrm{p}=0.589$ (table 1).

\section{HbA1c and glycaemic control}

Admission HbAlc was determined by either electronic patient records from the 1-month period prior to admission or, if unavailable, was taken at admission. Mean HbA1c levels were less overall in the survival group than in the non-survivors, $50.2 \mathrm{mmol} / \mathrm{mol}(\mathrm{SD} \pm 17.0)$ vs $60.8 \mathrm{mmol} /$ mol ( $\mathrm{SD} \pm 34.7$ ), but this was not statistically significant, $\mathrm{p}=0.284$. There was no statistical difference in outcome between patients who took insulin prior to admission and those with diet-controlled or tablet-controlled T2DM.

Weekly mean plasma glucose control was also assessed across the cohort of 71 (one individual's data were not fully available and therefore were omitted) and indicated no statistically significant difference between survivors and non-survivors. Overall, mean plasma glucose was deemed well controlled across both groups with mean glucose levels of $8.1 \mathrm{mmol} / \mathrm{L}(\mathrm{SD} \pm 1.9)$ and $8.9 \mathrm{mmol} / \mathrm{L}$ $(\mathrm{SD} \pm 2.3)$, respectively (table 2$)$.

Table 2 Mean plasma glucose control during the total length of stay

\begin{tabular}{llll}
\hline & \multicolumn{3}{l}{ Mean plasma glucose, mmol/L (SD) } \\
\cline { 2 - 4 } Week & Survived & Died & P value \\
\hline $1(n=71)$ & $8.0(2.2)$ & $9.0(2.7)$ & 0.125 \\
$2(n=66)$ & $8.3(2.2)$ & $9.3(2.4)$ & 0.132 \\
$3(n=47)$ & $7.9(1.9)$ & $9.1(2.4)$ & 0.063 \\
$4(n=32)$ & $8.0(1.8)$ & $9.3(2.7)$ & 0.112 \\
$5(n=19)$ & $8.2(2.1)$ & $8.8(2.2)$ & 0.618 \\
$6(n=7)$ & $7.9(2.3)$ & 11.0 & 0.267 \\
$7(n=5)$ & $7.4(1.7)$ & 8.7 & 0.551 \\
$8(n=2)$ & $7.4(1.8)$ & - & - \\
$9(n=1)$ & 8.4 & - & - \\
Entire stay $(n=71)$ & $8.1(1.9)$ & $8.9(2.3)$ & 0.083 \\
\hline
\end{tabular}


Table 3 Subcategories of vitamin D levels measured

\begin{tabular}{llll} 
& \multicolumn{2}{l}{ Frequency } & \\
\cline { 2 - 4 } $\begin{array}{l}\text { 25-hydroxyvitamin D } \\
\text { (nmol/L) }\end{array}$ & $\begin{array}{l}\text { Survived, } \\
\mathbf{n}=\mathbf{4 0}\end{array}$ & $\begin{array}{l}\text { Died, } \\
\mathbf{n = 1 1}\end{array}$ & P value \\
\hline$<25$ (deficient) & 17 & 8 & 0.131 \\
$\mathbf{2 6 - 5 0}$ (insufficient) & 15 & 3 & \\
$\mathbf{> 5 0}$ (adequate) & 8 & 0 & \\
\hline
\end{tabular}

$\mathrm{X}^{2}$ test.

\section{Vitamin D status}

A limited analysis of vitamin D status was possible as 51 (71\%) of the 72 patients included had a recorded vitamin D level either on admission or within 1 month of admission. If this was taken on admission, it was ensured that a correlating CRP was taken to aid interpretation. We subcategorised this measure into three groups as per UK national cut-offs for deficient, insufficient and sufficient serum concentration of 25-hydroxyvitamin D $(25(\mathrm{OH})$ D) (table 3). Overall, only $20 \%$ of patients had sufficient vitamin D levels. When comparing vitamin D levels across all three groups, no significant difference in mortality was observed, $\mathrm{p}=0.131$. Twenty-five individuals were deficient in vitamin $\mathrm{D}(<25 \mathrm{nmol} / \mathrm{L})$, of whom 8 died $(32 \%)$ and 17 survived. There was a similar rate of vitamin $\mathrm{D}$ insufficiency $(26-50 \mathrm{nmol} / \mathrm{L})$ in both groups (38\% of survivors, $27 \%$ of non-survivors). Furthermore, of those who died, none had sufficient levels of vitamin D prior to ICU admission.

Further analysis calculated the mean BMI of each group subcategorised by vitamin $\mathrm{D}$ status (table 4 ). This indicated those with deficiency or insufficiency were, on average, in the obese category. In comparison, the eight patients with sufficiency had an average BMI of $26.7 \mathrm{~kg}$ / $\mathrm{m}^{2}$, which is considered overweight. This may represent a trend between higher BMI and lower vitamin D levels, but this was not statistically significant $(p=0.238)$. We did not account for possible confounders known to impact COVID-19 outcomes, such as age, male sex, ethnicity or socioeconomic status, and this further limits the interpretation of this analysis.

\section{Analysis of variance}

We also analysed the correlation between CRP when serum concentration of $25(\mathrm{OH}) \mathrm{D}$ and found no significant correlation (figure 1).

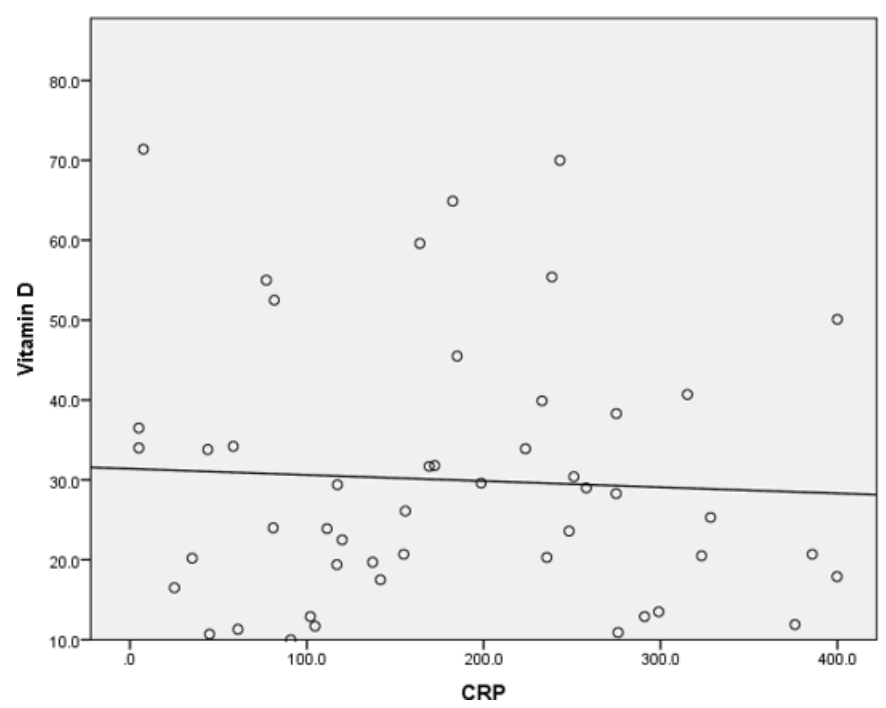

Figure 1 Vitamin D status $(25(\mathrm{OH}) \mathrm{D})$ in $\mathrm{nmol} / \mathrm{L}$ versus CRP (ng/L). Pearson correlation: $-0.053(p=0.718)$. 25(OH)D, 25-hydroxyvitamin D; CRP, C reactive protein.

\section{Other micronutrients}

In this group, selenium and zinc were measured in 33 of 72 $(46 \%)$ patients. For selenium, the mean serum level was $0.88 \mu \mathrm{mol} / \mathrm{L}$ (range $0.64-1.6 \mu \mathrm{mol} / \mathrm{L}$; normal reference range). For zinc, the mean serum level was $10.2 \mu \mathrm{mol} / \mathrm{L}$ (range 3.0-20.1 $\mathrm{mol} / \mathrm{L}$; normal reference range).

Folate and vitamin $\mathrm{B}_{12}$ levels were measured in 19 of $72(26 \%)$ patients. The mean vitamin $\mathrm{B}_{12}$ level was 803 ng/L (range 285-2000 ng/L; normal reference range). The mean serum folate was $5.4 \mu \mathrm{g} / \mathrm{L}$ (range $2.1-20 \mu \mathrm{g} / \mathrm{L}$; normal reference range).

\section{DISCUSSION}

\section{Patient demographics}

Our patient demographics for this audit aligned with the national picture in ICU at the time of data collection comparing with the Intensive Care National Audit \& Research Centre (ICNARC) data. This was comparable for age and gender and can be considered nationally representative in terms of ethnicity, with similar proportions of white, Asian and black patients ${ }^{1}$ (table 5).

A high percentage of our cohort were both older and men observing a higher case mortality rate in male patients, consistent with data from across China, Europe and the USA. ${ }^{14}$ This is reflective of higher risk demographics for COVID-19 corroborating with subsequent data establishing age and male gender as individual risk

Table 4 Mean BMI as per vitamin D status

\begin{tabular}{|c|c|c|c|c|}
\hline & \multicolumn{4}{|c|}{ 25-hydroxyvitamin D (nmol/L) } \\
\hline & $<25(n=25)$ & $26-50(n=18)$ & $>50(n=8)$ & $P$ value \\
\hline Mean BMI, kg/m² (SD) & $30.7(5.9)$ & $30.0(5.7)$ & $26.7(5.6)$ & 0.238 \\
\hline
\end{tabular}

BMI, body mass index. 
Table 5 Patient demographics versus ICNARC data

\begin{tabular}{|c|c|c|c|c|}
\hline \multirow[b]{2}{*}{ Characteristic } & \multicolumn{2}{|c|}{ Audit sample $(n=72)$} & \multicolumn{2}{|c|}{ ICNARC $(n=10624)$} \\
\hline & n (\%) & Median & n (\%) & Median \\
\hline Age & & $57-60$ & & 60 \\
\hline \multicolumn{5}{|l|}{ Gender (\%) } \\
\hline Male & \multicolumn{2}{|c|}{$75\left(66.7^{\star}\right)$} & \multicolumn{2}{|c|}{$70.2\left(58.3^{*}\right)$} \\
\hline Female & \multicolumn{2}{|c|}{$25\left(66.6^{\star}\right)$} & \multicolumn{2}{|c|}{$29.8\left(64.9^{\star}\right)$} \\
\hline \multicolumn{5}{|l|}{ Ethnicity } \\
\hline White & \multicolumn{2}{|l|}{68.1} & \multicolumn{2}{|l|}{66.2} \\
\hline Asian & \multicolumn{2}{|l|}{9.5} & \multicolumn{2}{|l|}{15.6} \\
\hline Black & \multicolumn{2}{|l|}{11.8} & \multicolumn{2}{|l|}{9.6} \\
\hline Mixed/other & \multicolumn{2}{|l|}{10.6} & \multicolumn{2}{|l|}{9.6} \\
\hline \multicolumn{5}{|c|}{$\mathrm{BMI}\left(\mathrm{kg} / \mathrm{m}^{2}\right)(\%)$} \\
\hline$<18.5$ & \multicolumn{2}{|c|}{0} & \multicolumn{2}{|c|}{0.8} \\
\hline $18.5-24.9$ & \multicolumn{2}{|c|}{$18.0\left(61.5^{\star}\right)$} & \multicolumn{2}{|c|}{$25.6\left(59.5^{\star}\right)$} \\
\hline $25.0-29.9$ & \multicolumn{2}{|c|}{$47.2\left(64.7^{\star}\right)$} & \multicolumn{2}{|c|}{$34.4\left(57.9^{*}\right)$} \\
\hline $30.0-40.0$ & \multicolumn{2}{|c|}{$29.2\left(66.7^{\star}\right)$} & \multicolumn{2}{|c|}{$31.4\left(63.6^{\star}\right)$} \\
\hline$\geq 40.0$ & \multicolumn{2}{|c|}{$5.6\left(100^{*}\right)$} & \multicolumn{2}{|c|}{$7.9\left(65.3^{*}\right)$} \\
\hline
\end{tabular}

*Survived critical care.

BMI, body mass index; ICNARC, Intensive Care National Audit \& Research Centre.

factors that adversely impact severity of disease. ${ }^{1}$ The median age observed in our audit was 57 years in the survival arm vs 60 years in the group that did not survive which was not statistically significant. However, this adds further data indicating increasing age as a risk factor in line with current literature. This is likely owing to higher rates of pre-existing comorbidities in older age noting cardiometabolic conditions as significant risk factors for COVID-19 which invariably are more prevalent in this population group. Equally factoring in physiological age-related decline in immunocompetence should be considered. ${ }^{15}$

Addressing the notion associating male sex with development of more severe disease has been observed in much larger studies analysed in a global meta-analysis which observed increased rate of ICU admissions in men seen as a proxy for disease severity. ${ }^{16}$ The explanation for these observations is likely multifactorial and complex and has recently been explored in terms of biological, behavioural and psychosocial factors. ${ }^{17}$ Genetic factors point towards an increased expression of ACE2 in men, as well as sex chromosome-related alterations in immune responses when compared with women. ${ }^{18} 19$ Behavioural and psychosocial factors are extensive relating to wellestablished literature linking men to increased risk via health behaviours such as increased tobacco and alcohol intake along with adherence to public health advice. ${ }^{1420}$

\section{Body mass index}

Our audit data observed a higher proportion of patients in the overweight than obese category in keeping with the trend seen in the ICNARC data. However, our data were less representative of individuals within BMI range $18.6-24.9 \mathrm{~kg} / \mathrm{m}^{2}$ and while acknowledging this as a limit there remained comparable trends to national data sets as further outlined. We noted that survivorship data were comparable across all BMI groups when cross-referencing to the ICNARC data set (table 4). We observed highest mortality for those in the obese class II BMI category $\left(35.0-39.9 \mathrm{~kg} / \mathrm{m}^{2}\right)$ but overall there was no statistically significant difference in mortality across BMI ranges, including the group in the severely obese category (BMI $\left.>40.0 \mathrm{~kg} / \mathrm{m}^{2}\right)$, who all survived.

While the results may be at odds with other recently published data on the relationship of severe obesity and disease severity, our data are in keeping with the ICNARC data and suggest obesity may increase risk of ICU admission but is not necessarily an individual marker for poor prognosis when used in isolation. ${ }^{421}$

First, there may have been substantial differences in the proportion of patients who died in the normal weight compared with overweight/obese categories, without there being an independent association between BMI and mortality, if controlling for the effect of metabolic and inflammatory derangements we know to be associated with worse disease outcomes. ${ }^{22}$ Data from the UK and China demonstrate higher mortality to be associated with poor glycaemic control, while hypertension has been found to be predictive of twofold higher mortality in observational data from a cohort in Wuhan. ${ }^{82}$ These data suggest that considering BMI status in isolation may not be an accurate predictor of death from COVID-19 and for any future data collection should ensure these confounding risk factors are considered and adjusted for accordingly.

The observation in patients in the highest BMI category is in line with previous meta-analyses showing inverse associations with mortality in ICU patients with severe obesity. ${ }^{24}$ The 'obesity paradox' phenomenon in critical care, including in patients with acute respiratory distress syndrome, describes how a lower mortality risk has been observed in obese versus nonobese patients. ${ }^{2526}$ This could be particularly relevant in those patients with COVID-19 requiring prolonged ICU admission. ${ }^{27}$

A number of physiological mechanisms may provide an explanation for these observations. Central obesity as measured by abdominal girth versus BMI status is a causal factor in atelectasis, which can have negative effects on pulmonary compliance, functional residual capacity and, ultimately, arterial oxygenation. ${ }^{28}$ Adipose tissue is highly metabolically active and obesity is associated with increased proinflammatory cytokines and adipokines that can lead to dysregulated immune reactions and drive inflammatory complications in critical illness. ${ }^{2}$ This indicates BMI alone may be too crude a measure to fully illicit the relationship between the implied metabolic/ immune effects differing adipose tissues exert in disease state but rather serves as a marker to highlight higher risk groups. 


\section{Glycaemic control and HbA1c}

Overall, there was a significantly higher proportion of patients with known T2DM in the group who died $(42 \%)$ compared with those who survived (19\%). On average, HbAlc levels were lower in those who survived but this difference did not reach statistical significance. This is in line with national findings whereby the largest UK-based population study found individuals with T2DM had increased odds of in-hospital death with COVID-19 (OR 2.03) compared with those without. ${ }^{8}$ It is therefore important to not only recognise this high-risk group in the critical care setting but equally understand how to mitigate some of this risk.

The pathophysiological interplay between COVID-19 and T2DM is not fully understood; however, it is well recognised that infection itself causes metabolic complications associated with adverse clinical outcomes. COVID-19 can precipitate and worsen acute metabolic complications associated with both T1DM and T2DM, such as diabetic ketoacidosis and hyperglycaemia, which may impact mortality rates in this group. ${ }^{29}$ Mechanistic data also support this relationship, whereby it has been suggested that hyperglycaemia may upregulate expression of ACE2 receptor, which is understood to be the entry point for the SARS-CoV-2 into cells. ${ }^{30}$ Other proposed mechanisms include altered ACE2 activity, chronic inflammation, accelerated endothelial damage and hypercoagulable states. All of these might accelerate pre-existing end organ damage relating to diabetes, and perhaps even more so in individuals with poorer longterm diabetic control. ${ }^{5}$

Targets for glycaemic control within the ICU setting are controversial with no definitive consensus on what constitutes 'optimal control'. There is agreement when understanding the detrimental effects of both hyperglycaemia and hypoglycaemia but not as to what targets most optimally minimise this.

In this audit, mean plasma glucose levels were within a well-controlled range for the duration of both groups of ICU stay: $8.1 \mathrm{mmol}$ (SD 1.9) in the survival group and $8.9 \mathrm{mmol}(\mathrm{SD} \pm 2.3)$ in the non-survivors $(\mathrm{p}=0.083)$ with an overall mortality rate of $33 \%$. HbA1c levels were lower overall in the survival group $(50.2 \mathrm{mmol} / \mathrm{mol}$ vs 60.8 $\mathrm{mmol} / \mathrm{mol}$ ), but this association did not reach statistical significance. NHS England data comparing COVID-19 outcomes in patients with higher HbAlc $(>86 \mathrm{mmol} / \mathrm{mol})$ versus those with $\mathrm{HbAlc}$ between 48 and $53 \mathrm{mmol} / \mathrm{mol}$ found mortality increased with higher HbAlc in patients with both T1DM and T2DM. ${ }^{8}$ In a retrospective analysis of patients with T2DM in Wuhan, maintaining in-hospital glycaemic variability within $3.9-10.0 \mathrm{mmol} / \mathrm{L}$ was associated with a significant reduction in adverse outcomes and death (1\% vs $11 \%) \cdot{ }^{23}$ These observations suggest that glycaemic control should be an important consideration in COVID-19 illness and this should continue to garner attention as dexamethasone, with its side effect of hyperglycaemia, is included in standard treatment protocols of inpatients with COVID-19.

\section{Vitamin D status}

In our cohort, we observed a negative correlation between vitamin $\mathrm{D}$ status and $\mathrm{BMI}$, though this association did not reach statistical significance. Beyond the association with BMI, vitamin D deficiency $(<25 \mathrm{nmol} / \mathrm{L})$ was seemingly associated with increased mortality in the group, whereby 8 of the 25 patients with vitamin $\mathrm{D}$ deficiency died. Conversely, all patients with vitamin D status $>50$ $\mathrm{nmol} / \mathrm{L}$ survived, posing the question as to whether an improved vitamin D status contributes to better outcomes in the ICU.

Multiple analyses of UK Biobank data have found a higher frequency of vitamin D deficiency in patients with COVID-19 when compared with population-based controls. ${ }^{31}$ However, these data must be interpreted with caution based on a lack of statistical significance when adjusted for confounding factors and also due to data being largely historical. A study of healthcare workers in the UK observed $72 \%$ of those with vitamin D deficiency tested positive for COVID-19 compared with $51 \%$ of those without vitamin D deficiency. ${ }^{32}$ Elsewhere data from Israel have shown vitamin D levels estimated between 2010 and 2019 to correlate strongly with COVID-19 incidence. ${ }^{33} \mathrm{In}$ the USA, strong associations have been found between lower 25(OH)D levels and increased rate of SARS-CoV-2 positivity, even after adjusting for confounders. ${ }^{34}$ These studies did not include data on COVID-19 severity.

The association of low vitamin D status with higher BMI could be explained by physiological, behavioural, genetic and even geographical determinants. ${ }^{35-38}$ Physiological mechanisms include increased storage in adipose tissue and altered metabolism, due to reduced metabolite activation secondary to lower levels of enzymes in adipose tissue for hydroxylation. Behavioural factors have been particularly noteworthy during the pandemic, particularly in those institutionalised and/or shielding, who as a result receive less sun exposure and therefore obtain less vitamin D synthesis. Genetic and geographical determinants of vitamin D status based on latitude and ethnicity have been well described elsewhere. ${ }^{38}$

Vitamin D deficiency in critical illness is known to be associated with poorer outcomes and supplementation has largely been demonstrated as safe; however, defining optimum target ranges, clinical assays and measurable metabolites has proven difficult. ${ }^{39}$ Of the extraskeletal functions of vitamin $\mathrm{D}$, potential immunomodulatory effects have received attention in this patient group. Higher vitamin D status is thought to play a role in modulating the inflammatory response, whereas low levels appear to be associated with the activation of inflammatory processes. ${ }^{39}$ The relationship between vitamin $\mathrm{D}$ and inflammation could be particularly relevant in critically ill patients. ${ }^{40}$ For example, the activation of vitamin $\mathrm{D}$ in the lung has the potential to induce specific antimicrobials and attenuate inflammatory cytokines in response to viruses, which is thought to have a role in the case of sepsis derived from lung infection. ${ }^{41}$ 
Retrospective meta-analyses from American and European cohorts have indicated low vitamin $\mathrm{D}$ status to be associated with higher rates of ICU admission in patients with COVID-19. Similar to trends observed in our analysis, this translated to poorer prognosis, while also highlighting significantly lower vitamin D status in ICU compared with patients receiving ward-based care. ${ }^{42}$ Furthermore, in a smaller observational study from Italy, a survival analysis of patients with COVID-19 admitted to a respiratory intermediate care unit found severe vitamin $\mathrm{D}$ deficiency to be a predictor of mortality. Those with vitamin D levels $<25$ $\mathrm{nmol} / \mathrm{L}$ had a $50 \%$ probability of mortality, compared with a $5 \%$ risk in those with levels $\geq 25 \mathrm{nmol} / \mathrm{L}{ }^{43}$ It is of course important to recognise limitations within these studies in terms of sample size and methodology. However, in the early stages of this pandemic, the role of these observations is to provide a basis on which more rigorous scientific trials can be designed to help establish causal links between such factors and the pathophysiology of SARS-CoV-2.

This increasing depth of observational data has resulted in calls for more clinical trials to evaluate a potential therapeutic role of vitamin D in COVID-19. The results of the first of these pilot studies have recently been published from Spain, which assessed oral supplementation of high-dose calcifediol $(25(\mathrm{OH}) \mathrm{D})$ alongside combination drug therapy in patients with COVID-19, demonstrating a reduction in critical care admissions in those supplemented. ${ }^{44}$ Even in the absence of definitive evidence from interventional trials, since avoiding vitamin $\mathrm{D}$ deficiency is already an accepted public health priority in the UK, a pragmatic approach would be to test and safely treat for suboptimal vitamin D levels in all patients with COVID19. There is a clear demand for clinical trials investigating vitamin D supplementation in the prevention of COVID-19 in patients with COVID-19 at the earliest stage of infection, for example, at the point of receiving a positive SARS-CoV-2 test result in the community.

\section{Other micronutrients}

In this audit, we collected all micronutrient data that were measured in this cohort. Serum selenium and zinc were measured in only $46 \%$ of patients, and vitamin $B_{12}$ and folate measured in even fewer, despite these being important micronutrients for immune function and their measurements being commonly available laboratory tests.

The latest clinical guidelines from the European Society for Clinical Nutrition and Metabolism on the nutritional management of patients with COVID-19 highlight the importance of ensuring sufficient levels of essential micronutrients to "potentially reduce disease negative impact'. ${ }^{3}$ Specifically mentioned are vitamins $\mathrm{C}$, $\mathrm{D}, \mathrm{A}, \mathrm{E}, \mathrm{B}_{6}$ and $\mathrm{B}_{12}$, and zinc, selenium and iron, whose potential importance is inferred from existing research into their roles in immune function and outcomes in other infectious diseases. However, there remains a lack of evidence from robust interventional studies regarding micronutrient management in COVID-19. Furthermore, there is no evidence that supplementation is beneficial to patients whose levels are already replete.

\section{Other clinical and research considerations}

One harsh reality is that, since the majority of patients hospitalised with COVID-19 will be elderly and with other comorbidities, many will not be offered more intensive treatments available in ICU. Therefore, clinical audit and trials from outside the ICU setting are also crucially important. From a practical perspective, it would be wise to frame sensible clinical nutrition interventions such as ensuring micronutrient adequacy as the responsibility of all healthcare providers caring for patients with COVID-19 and people at risk.

In the absence of robust interventional data, the nutritional management of patients with COVID-19 in critical care remains consensus guided and based on foundational principles. Broadly, these are: routine screening for malnutrition; supplementation for insufficiency and deficiency; a 'food first' approach, including dietary modification for nutritional content and texture; oral nutritional supplementation, and escalation to enteral, then parenteral, nutrition where appropriate. ${ }^{3}$ Indeed, ensuring adequacy of a single micronutrient is not helpful, and a holistic approach to nutritional care, involving a multidisciplinary team of dietitian, nurse, doctor and other allied health professionals, is key. An important yet unanswered question is how often clinicians should investigate micronutrient levels in anticipation of increased requirements, and the presence of systemic inflammation. What is accepted is that management of nutrition in critical care for patients with COVID-19 must be individualised on a case-by-case basis. ${ }^{45}$

\section{Limitations}

The findings of this retrospective analysis are time and location specific with limitations in terms of generalisability across different geographical and demographic contexts. We have referenced national and international data sets for comparisons to guide applicability and generalisability to similar regions. The small sample size and retrospective nature of the data collection were never powered to answer a specific clinical question. Logistical barriers such as unprecedented workload and cumbersome personal protective equipment requirements likely impacted accuracy of some measurements, particularly accurate weight and height. Indeed, for many parameters, for example vitamin $\mathrm{D}$, a proportion of patients had no measurement recorded, which poses a high risk of bias to the results in such a small sample. The gaps in our data also made it difficult to account for probable confounders (age, sex, ethnicity, socioeconomic status) when statistically analysing associations between parameters and outcomes. Shared access to electronic community healthcare records provided access to recently recorded data where these were not immediately available, for example, height and weight, vitamin D level and HbA1c. Selection bias was minimised by including all individuals during the 


\section{Implications for clinical practice}

- Baseline nutritional measurements, including capillary glucose, haemoglobin A1c (if type 2 diabetes mellitus present) and serum micronutrients such as vitamin $\mathrm{D}$, selenium and zinc can be readily made in a critical care context in this pandemic.

- Obtaining accurate measures of weight and correlating with body mass index may help as part of risk stratification when assessing clinical prognosis.

- This intensive care unit department achieved adequate control of capillary blood glucose levels despite significant logistical challenges and with increasing use of dexamethasone may warrant further investigation.

- Vitamin D status in the critically ill patient is easily obtainable and treatment to address deficiency remains in keeping with consensus guidelines

time period, but information bias could not be fully mitigated against.

Collectively, these limitations also restrict our ability to establish any causality (consistency, temporality, specificity, coherence, experiment and analogy) to define relationships between nutritional measures and COVID-19 outcomes. If more nutritional data from similar settings are reported, this could allow judgements of consistency in relationships across studies in the future.

\section{CONCLUSIONS}

This was a single-centre service evaluation of clinical and biochemical data relating to nutritional status from patients with COVID-19 admitted to ICU during the first peak of the 2020 pandemic. We were able to measure markers of nutritional status such as serum micronutrients, vitamin D levels and blood glucose more accurately when compared with anthropometric measures such as height and weight, owing to the unstable nature of this patient group and increased clinical pressures during this phase of the pandemic. There was a high prevalence of T2DM and vitamin D insufficiency/deficiency in this ICU cohort and T2DM was more prevalent in those who died from COVID-19. Of these, low vitamin D is more straightforward to remedy in the acute setting, which supports the notion that active detection and correction of vitamin $\mathrm{D}$ deficiency is warranted in patients with, and at greater risk of, COVID-19. However, our data suggest that BMI in isolation may not be an accurate predictor of mortality in COVID-19 without consideration of a fuller clinical picture.

With our evolving understanding of COVID-19 and the interplay with nutrition, this presented audit helps to signpost to important markers for consideration and advocates for better standardisation in nutrition-related measures for future audits. Alongside BMI, further anthropometric data collection may help refine our risk stratification and subsequent public health advice. Equally by measuring HbAlc as routine would also help to risk stratify patients in and outside of the critical care setting as steroid therapy is common practice which subsequently impacts glycaemic control and may help to mitigate additional complications. Further nutritional measures of micronutrient status specifically a panel implicated in immune function and inflammatory mediation (vitamins C, D, E, zinc and selenium) could help to identify individuals at greater risk. This would also help to establish if simple, low-risk interventions such as targeted micronutrient supplementation confer clinical benefit and could help to explore interventions which are not exclusive to the critical care setting. Furthermore, prospective data capture, larger observational studies and robust, well-designed interventional trials are required to better establish best practice for clinical nutrition approaches and the management of nutritional and metabolic comorbidities in patients with COVID-19.

\section{Author affiliations}

${ }^{1}$ Hammersmith Hospital, Imperial College Healthcare NHS Trust, London, UK ${ }^{2}$ NNEdPro Global Centre for Nutrition and Health, St John's Innovation Centre, Cambridge, UK

${ }^{3}$ Clinical Sciences and Nutrition, University of Chester, Chester, UK

${ }^{4}$ School of Biomedical Sciences, Ulster University-Coleraine Campus, Coleraine, UK

${ }^{5}$ School of Humanities and Social Sciences, University of Cambridge, Cambridge, UK ${ }^{6}$ School of Public Health, Imperial College London, London, UK

Twitter Timothy Eden @DrTimothyEdenRD and Shane McAuliffe @ShaneMacZ

Acknowledgements We would like to thank the NNEdPro COVID-19 Taskforce and the wider clinical team at Imperial College Healthcare NHS Trust for the support during the data collection process and the write up of this service evaluation.

Contributors TE, JN and SR planned the above service evaluation and determined the data collection. TE and JN collected the relevant data and JN and SR provided the statistical analysis. TE, SM and DC contributed to the report writing and discussion of this service evaluation. SR supervised the undertaking of the work and contributed to the overall revisions prior to submission by TE.

Funding The authors have not declared a specific grant for this research from any funding agency in the public, commercial or not-for-profit sectors.

Competing interests None declared.

Patient consent for publication Not required.

Provenance and peer review Not commissioned; externally peer reviewed by Marcello Scopazzini, NHS Lothian, Edinburgh, UK.

Data availability statement All data relevant to the study are included in the article or uploaded as supplemental information. All relevant data and statistical analyses have been included within the submission. For further information and additional data, please contact the first author Timothy Eden (timothy.eden@nhs. net).

Supplemental material This content has been supplied by the author(s). It has not been vetted by BMJ Publishing Group Limited (BMJ) and may not have been peer-reviewed. Any opinions or recommendations discussed are solely those of the author(s) and are not endorsed by BMJ. BMJ disclaims all liability and responsibility arising from any reliance placed on the content. Where the content includes any translated material, BMJ does not warrant the accuracy and reliability of the translations (including but not limited to local regulations, clinical guidelines, terminology, drug names and drug dosages), and is not responsible for any error and/or omissions arising from translation and adaptation or otherwise.

Open access This is an open access article distributed in accordance with the Creative Commons Attribution Non Commercial (CC BY-NC 4.0) license, which permits others to distribute, remix, adapt, build upon this work non-commercially, and license their derivative works on different terms, provided the original work is properly cited, appropriate credit is given, any changes made indicated, and the use is non-commercial. See: http://creativecommons.org/licenses/by-nc/4.0/. 
ORCID iD

Shane McAuliffe http://orcid.org/0000-0002-7166-4299

\section{REFERENCES}

1 Intensive Care National Audit and Research Centre (ICNARC). ICNARC report on COVID-19 in critical care 31 July 2020, 2020.

2 Calder PC. Nutrition, immunity and COVID-19. BMJ Nutr Prev Health 2020;3:74-92.

3 Barazzoni R, Bischoff SC, Breda J, et al. ESPEN expert statements and practical guidance for nutritional management of individuals with SARS-CoV-2 infection. Clin Nutr 2020;39:1631-8.

4 Simonnet A, Chetboun M, Poissy J, et al. High prevalence of obesity in severe acute respiratory syndrome Coronavirus-2 (SARS-CoV-2) requiring invasive mechanical ventilation. Obesity 2020;28:1195-9.

5 Williamson EJ, Walker AJ, Bhaskaran K, et al. Factors associated with COVID-19-related death using OpenSAFELY. Nature 2020;584:430-6.

6 Steele RM, Finucane FM, Griffin SJ, et al. Obesity is associated with altered lung function independently of physical activity and fitness. Obesity 2009;17:578-84.

7 Peng YD, Meng K, Guan HQ, et al. [Clinical characteristics and outcomes of 112 cardiovascular disease patients infected by 2019nCoV]. Zhonghua Xin Xue Guan Bing Za Zhi 2020;48:450-5.

8 Barron E, Bakhai C, Kar P, et al. Associations of type 1 and type 2 diabetes with COVID-19-related mortality in England: a wholepopulation study. Lancet Diabetes Endocrinol 2020;8:813-22.

9 Murad MH, Coburn JA, Coto-Yglesias F, et al. Glycemic control in non-critically ill hospitalized patients: a systematic review and metaanalysis. J Clin Endocrinol Metab 2012;97:49-58.

10 Zittermann A, Pilz S, Hoffmann $\mathrm{H}$, et al. Vitamin D and airway infections: a European perspective. Eur J Med Res 2016;21:1-10.

11 NDNS: results from Years 1 to 4 (combined) - GOV.UK [Internet]. PHE and FSA (2014b) Statistical Press Notice: National Diet and Nutrition Survey: results from years 1, 2, 3 and 4 combined (2008/09 - 2011/12). Public Health England and Food Standards Agency. [cited 2021 Jan 24]. Available: https://www.gov.uk/government/ statistics/national-diet-and-nutrition-survey-results-from-years-1-to4-combined-of-the-rolling-programme-for-2008-and-2009-to-2011and-2012

12 Pugach IZ, Pugach S. Strong correlation between prevalence of severe vitamin $D$ deficiency and population mortality rate from COVID-19 in Europe. Wien Klin Wochenschr 2021;133:403-5.

13 Martineau AR, Jolliffe DA, Hooper RL, et al. Vitamin D supplementation to prevent acute respiratory tract infections: systematic review and meta-analysis of individual participant data. BMJ 2017;356:i6583.

14 Baker P, White A, Morgan R. Men's health: COVID-19 pandemic highlights need for overdue policy action. Lancet 2020;395:1886-8.

15 Accardi G, Caruso C. Immune-Inflammatory responses in the elderly: an update. Immun Ageing 2018;15:11 https://immunityageing. biomedcentral.com/articles/

16 Peckham $\mathrm{H}$, de Gruijter NM, Raine $\mathrm{C}$, et al. Male sex identified by global COVID-19 meta-analysis as a risk factor for death and ITU admission. Nat Commun 2020;11:1-10.

17 Griffith DM, Sharma G, Holliday CS, et al. Men and COVID-19: a biopsychosocial approach to understanding sex differences in mortality and recommendations for practice and policy interventions. Prev Chronic Dis 2020;17:E63.

18 Sama IE, Ravera A, Santema BT, et al. Circulating plasma concentrations of angiotensin-converting enzyme 2 in men and women with heart failure and effects of renin-angiotensin-aldosterone inhibitors. Eur Heart J 2020;41:1810-7.

19 Sharma G, Volgman AS, Michos ED. Sex differences in mortality from COVID-19 pandemic. JACC: Case Reports 2020;2:1407-10.

20 De La Vega R, Barquin RR, Boros S. Could attitudes toward COVID-19 in Spain render men more vulnerable than women? [cited 2020 Oct 25], 2020. Available: https://psyarxiv.com/dyxqn/

21 Lemyze M, Courageux N, Maladobry T, et al. Implications of obesity for the management of severe coronavirus disease 2019 pneumonia. Crit Care Med 2020;48:e761-7.
22 Finucane FM, Davenport C, Coronavirus DC. Coronavirus and obesity: could insulin resistance mediate the severity of Covid-19 infection? Front Public Health 2020;8:184.

23 Zhu L, She Z-G, Cheng X, et al. Association of blood glucose control and outcomes in patients with COVID-19 and pre-existing type 2 diabetes. Cell Metab 2020;31:1068-77.

24 Hogue CW, Stearns JD, Colantuoni E, et al. The impact of obesity on outcomes after critical illness: a meta-analysis. Intensive Care Med 2009;35:1152-70.

25 De Jong A, Molinari N, Sebbane M, et al. Feasibility and effectiveness of prone position in morbidly obese patients with ARDS: a case-control clinical study. Chest 2013;143:1554-61.

26 De Jong $A$, Jung $B$, Chanques $G$, et al. Obesity and mortality in critically ill patients: another case of the Simpson paradox? Chest 2012;141:1637-8.

27 Whittle J, Molinger J, MacLeod D, et al. Persistent hypermetabolism and longitudinal energy expenditure in critically ill patients with COVID-19. Crit Care 2020;24:581.

28 De Jong A, Chanques G, Jaber S. Mechanical ventilation in obese ICU patients: from intubation to extubation. Crit Care 2017;21:63.

29 Apicella M, Campopiano MC, Mantuano M, et al. COVID-19 in people with diabetes: understanding the reasons for worse outcomes. Lancet Diabetes Endocrinol 2020;8:782-92.

30 Muniyappa R, Gubbi S. COVID-19 pandemic, coronaviruses, and diabetes mellitus. Am J Physiol Endocrinol Metab 2020;318:E736-41.

31 Hastie CE, Pell JP, Sattar N. Vitamin D and COVID-19 infection and mortality in UK Biobank. Eur J Nutr 2021;60:545-8.

32 Faniyi AA, Lugg ST, Faustini SE. Vitamin D status and seroconversion for COVID-19 in UK healthcare workers who isolated for COVID-19 like symptoms during the 2020 pandemic. medRxiv 2020.

33 Israel A, Cicurel A, Feldhamer I. The link between vitamin D deficiency and Covid-19 in a large population. medRxiv 2020.

34 Kaufman HW, Niles JK, Kroll MH, et al. SARS-CoV-2 positivity rates associated with circulating 25-hydroxyvitamin D levels. PLoS One 2020;15:e0239252.

35 Pourshahidi LK. Vitamin D and obesity: Current perspectives and future directions. In: Proceedings of the Nutrition Society [Internet. Cambridge University Press, 2015: 74. 115-24.

36 Griffin TP, Wall D, Blake L. Vitamin D status of adults in the community, in outpatient clinics, in hospital, and in nursing homes in the West of Ireland. Journals Gerontol Ser A [Internet] 2020 https:// pubmed.ncbi.nlm.nih.gov/31942614/

37 Vimaleswaran KS, Berry DJ, Lu C, et al. Causal relationship between obesity and vitamin D status: bi-directional Mendelian randomization analysis of multiple cohorts. PLoS Med 2013;10:e1001383.

38 Kohlmeier M. Avoidance of vitamin D deficiency to slow the COVID-19 pandemic. BMJ Nutr Prev Health 2020;3:67-73.

39 Nair P, Venkatesh B, Center JR. Vitamin D deficiency and supplementation in critical illness-the known knowns and known unknowns. Crit Care 2018;22:276.

40 Amrein K, Papinutti A, Mathew E, et al. Vitamin D and critical illness: what endocrinology can learn from intensive care and vice versa. Endocr Connect 2018;7:R304-15.

41 Hansdottir S, Monick MM, Hinde SL, et al. Respiratory epithelial cells convert inactive vitamin $\mathrm{D}$ to its active form: potential effects on host defense. J Immunol 2008;181:7090-9.

42 Munshi R, Hussein MH, Toraih EA, et al. Vitamin D insufficiency as a potential culprit in critical COVID-19 patients. J Med Virol 2021;93:733-40.

43 Carpagnano GE, Di Lecce V, Quaranta VN, et al. Vitamin D deficiency as a predictor of poor prognosis in patients with acute respiratory failure due to COVID-19. J Endocrinol Invest 2021;44:765-71.

44 Entrenas Castillo M, Entrenas Costa LM, Vaquero Barrios JM, et al. "Effect of calcifediol treatment and best available therapy versus best available therapy on intensive care unit admission and mortality among patients hospitalized for COVID-19: A pilot randomized clinical study". J Steroid Biochem Mol Biol 2020;203:105751.

45 Berger MM. Nutrition and micronutrient therapy in critical illness should be individualized. JPEN J Parenter Enteral Nutr 2020;44:1380-7 